\title{
STRANGELETS IN TERRESTRIAL ATMOSPHERE
}

\author{
Shibaji Banerjee ${ }^{a}$ 凹, Sanjay K. Ghosh ${ }^{a}$, Sibaji Raha $\left.{ }^{a}\right]$ and Debapriyo \\ Syam $^{b}$
}

\author{
(a) Department of Physics, Bose Institute, \\ 93/1, A. P. C. Road, Calcutta 700 009, INDIA
}

(b) Department of Physics, Presidency College, 86/1, College Street, Calcutta 700 073, INDIA

\begin{abstract}
A new dynamical model for the propagation of strangelets through the terrestrial atmosphere is proposed.
\end{abstract}

Strangelets are small lumps of strange quark matter (SQM) which consist of roughly equal numbers of up, down and strange quarks. Since the suggestion of Witten[1] in 1984 that these strangelets, and not ordinary nuclear matter, represent the true ground state of QCD, this area of research has expanded considerably. The existence of stable or metastable lumps of SQM would have numerous implications for physics and astrophysics. Most importantly, they can account for the cosmological dark matter problem to a large extent, if not entirely [2]. Moreover, the mere existence of strangelets may be one of the most perfect evidences for the QGP phase transition that is believed to have occurred in the early universe or inside neutron stars.

\footnotetext{
${ }^{1}$ email: phys@boseinst.ernet.in

${ }^{2}$ email: phys@boseinst.ernet.in

${ }^{3}$ email: sibaji@boseinst.ernet.in
} 
To establish the existence of strangelets, it is, of course, necessary to detect them. The obvious place to search for them would be in the cosmic rays. To this end, it is necessary to understand the propagation of strangelets through the earth's atmosphere, as these would have to traverse the whole atmospheric depth before arriving at the detectors. In this letter, we attempt to provide a realistic dynamical model which describes the propagation of strangelets.

The fact that SQM is absolutely stable does not contradict the ordinary experience that matter is, for the most part, made of ordinary nuclear matter. This is because, in order for ordinary matter to change to SQM, a large number of $u$ and $d$ quarks need to change to $s$ quarks, which would require a very high order weak interaction and therefore can be considered to be highly improbable [1]. On the other hand, the existence of ordinary nuclear matter shows that quark matter consisting of only $u$ and $d$ quarks is unstable [1]. Introduction of a third flavour reduces the energy relative to a two-flavour system since another Fermi well is now present and this makes the system stable. In the earlier works [3], calculations along the lines of Fermi gas model seemed to suggest that although strangelets with high atomic numbers would be absolutely stable, the same could not be said for strangelets with low A; the surface effect which must be taken into account for small strangelets would make the energy per baryon increase with decreasing baryon number, turning the SQM unstable. In later calculations [ [4, 5], the Fermi gas model was replaced by the spherical MIT hadronic bag models and the stability of strangelets with low A was examined in the light of this model. The works done by various authors [6, 7] tell us that for low $s$-quark masses, shell-like structures occur for $\mathrm{A}=6,18,24,42,54,60,84,102$ etc. These values may change somewhat with the change in the strangeness fraction [6, 7].

Strangelets are expected to possess a small positive electric charge. They would have been neutral, if the ground state composition consisted of equal numbers of quarks of the three types of quark flavours, which is the most favourable state. Actu- 
ally, however, the $s$ quark is heavier than the other two flavours and so their number is slightly less than that of the $u$ or $d$ quarks. Hence the fortuitous cancellation $\frac{2}{3} n_{u}-\frac{1}{3} n_{d}-\frac{1}{3} n_{s}$ does not occur exactly, as a result of which we are left with a small residual positive charge. It can also be argued from mere experience that they cannot be negatively charged because although overall charge neutrality can be maintained by covering them up by a cloud of positrons, they will eat up every piece of ordinary matter they find in their path since it is energetically favorable for nuclear matter to convert to SQM. (For a recent survey of the physics of SQM, see [8].) On the other hand, a small positive charge would be helpful, as in that case, ordinary nuclei will be electrostatically repelled when the strangelets are moving slowly; when they move fast enough (highly energetic), nothing can prevent them from absorbing neutrons and becoming more and more tightly bound.

It is generally assumed that the strangelets which come to the upper layer of the atmosphere have baryon number $A \sim 1000$ or more. This assumption is usually made on the basis of the following experimental and theoretical considerations. Some of the reports which suggest direct candidates for SQM are tabulated below.

\begin{tabular}{|l|c|c|}
\hline Event & Mass & Charge \\
\hline Counter experiment [9] & $A \sim 350-450$ & 14 \\
Exotic Track [10 & $A \sim 460$ & 20 \\
Price's Event [1] & $A>1000$ & 46 \\
Balloon Experiments [12, 13] & $A \sim 370$ & 14 \\
\hline
\end{tabular}

The theoretical tip is usually taken from the generally accepted mass number for strangelets which is given in the above paragraph. It is often remarked that that the observation of these candidates at such large atmospheric depths $\left(\sim 500 \mathrm{~g} / \mathrm{cm}^{2}\right)$ requires unusual penetrability of these lumps which means that their cross sections should be very small and hence the geometric size much smaller than typical nuclear 
size. This, however, is not borne out by the models of SQM where the density of SQM is seen to be not much larger than ordinary nuclear matter [1].

Wilk et al. 114, 15, 16, 17] proposed a mechanism by which the strangelets are able to cover great atmospheric depths. They assumed that the mass and hence the cross sections of the strangelets incident on the upper layers of the atmosphere with initial masses of the order of $10^{3}$ a.m.u. decrease rapidly due to their collisions with air molecules in which a mass equal to that of the nucleus of an atmospheric atom is ripped off from the strangelet in every encounter. They also proposed that there should exist a critical mass $m_{c r i t}$ such that when the mass of the strangelet evolving out of an initially large strangelet drops below that, it simply evaporates into neutrons and that is what would fix the lower limit of the altitude upto which a strangelet would be able to penetrate.

Let us recapitulate the basic conclusions that can be derived from the earlier works. Firstly, strangelets observed at the mountain altitudes typically have mass around 300 to 450 and charge between 10 to 20 . But the experimental results obtained till date are inconclusive and hence they do not impose a strict bound on the mass and charge of strangelets that can be observed in future experiments. Secondly, although the correlation between penetrability and geometric cross sections is usually valid for ordinary nuclei, the same cannot be easily extrapolated to the case of strangelets since these are very tightly bound massive objects and are not expected to break up as a result of nuclear collisions. Indeed, in a typical interaction between a strangelet and the nucleus of an atmospheric atom, it is more probable for the strangelet to absorb neutrons so that the colliding nucleus, and not the strangelet, is likely to break up most of the time. Hence the scheme proposed in [14], namely that the mass of a strangelet decreases in every encounter, seems to be unrealistic. We, in this letter, propose an alternative scheme based on the following premises :

1. The collision of a lump of SQM with ordinary matter results in the absorption of the neutrons from the colliding nucleus, as a result of which the mass of the 
strangelet increases in every collision and it becomes more tightly bound.

2. The initial masses of the strangelets are assumed to be small in order to obtain final baryon numbers which are nearly equal to the observed ones at mountain altitudes. The discussion above indicates that it is quite possible to have stable lumps of SQM with low mass numbers. This would also facilitate a somewhat larger flux in the cosmic rays.

3. The speed, and hence the kinetic energy of these particles, must be such that they would arrive at a distance of $25 \mathrm{~km}$ above the sea level, surmounting the geomagnetic effects. We start with such an altitude since the atmospheric density above $25 \mathrm{~km}$ is low enough to be neglected. The charge of the strangelet is also fixed by this assumption, corresponding to a certain strangeness fraction.

The simple assumptions proposed above give a picture more or less in accord with the observation of the propagation of the strangelets in the atmosphere, which can give useful indications of the type of things to be expected in an actual experiment. The description of the model is given next. We consider a situation in which a strangelet with a low baryon number enters the upper layers $(\sim 25 \mathrm{Km}$ from the sea level) of the atmosphere. To arrive at this point, a charged particle must possess a speed determined by the formula (see, e.g, [18]).

$$
\frac{p c}{Z e} \geq \frac{M}{r_{o}^{2}} \frac{\cos ^{4} \vartheta}{\left(\sqrt{1+\cos ^{3} \vartheta}+1\right)^{2}}
$$

where $M$ is the magnetic dipole moment of the Earth, $r_{o}$ the radius of the Earth and $\vartheta$ is the (geomagnetic) latitude of the point of observation $\left(\sim 30^{\circ}\right.$, which might represent a location in north eastern India). $p$ and $Z e$ represent the momentum and charge, respectively, of the particle. The magnetic field of the Earth is taken to be equivalent to that due to a magnetic dipole of moment $M=8.1 \times 10^{22} \mathrm{~J} / T$, located near the centre of the earth, the dipole axis pointing south. We have fixed the mass, 
initial speed and charge to be $64 \mathrm{amu} 6.6 \times 10^{7} \mathrm{~m}$ per sec and 2 (electron charge), respectively, at the initial altitude of $25 \mathrm{~km}$, for the purpose of the present work.

In the course of its journey, the strangelet comes in contact with air molecules, mainly $N_{2}$. During such collisions, the strangelet absorbs neutrons from some of these molecules, as a result of which it becomes more massive. The effect of such encounters is summarized in the formula

$$
\frac{d m_{S}}{d h}=\frac{f \times m_{N}}{\lambda}
$$

where $m_{S}$ is the mass of the strangelet, $m_{N}$ the total mass of the neutrons in the atmospheric atom, $\lambda$ the mean free path of the strangelet in the atmosphere and $h$ the path length traversed. (It should be emphasized here that the strangelets would preferentially absorb neutrons, as protons would be coulomb repelled. Nonetheless, some protons could still be absorbed initially when the relative velocity between the strangelet and the air molecule is large, leading to increase in both the mass and the charge of the strangelet. We do not address this issue in the present work for the sake of simplicity, although it can be readily seen that the rate of increase in mass would obviously be faster than that in charge.) In the above equation, $\lambda$ depends both on $h$, which determines the density of air molecules and the instantaneous mass of the strangelet, which relates to the interaction cross section. The mean free path decreases as lower altitudes are reached since the atmosphere becomes more dense and the collision frequency increases. Finally the factor $f$ determines the fraction of neutrons that are actually absorbed out of the total number of neutrons in the colliding nucleus. The expression for this factor has been determined by geometric considerations [19] and is given by

$$
f=\frac{3}{4}(1-\nu)^{1 / 2}\left(\frac{1-\mu}{\nu}\right)^{2}-\frac{1}{3}\left[3(1-\nu)^{1 / 2}-1\right]\left(\frac{1-\mu}{\nu}\right)^{3}
$$

In eqn(3), $\mu=\frac{b}{R_{1}+R_{2}}$ and $\nu=\frac{R_{1}}{R_{1}+R_{2}}$ where $b$ is the impact parameter. $R_{1}$ and $R_{2}$ are the radii of the strangelet and the nucleus of the atmospheric atom, respectively. $f$ is 
initially small but grows larger and reaches the limiting value 1 when the strangelet grows more massive.

The above considerations lead us to a set of differential equations of the form

$$
\frac{d \vec{v}}{d t}=-\vec{g}+\frac{q}{m_{S}}(\vec{v} \times \vec{B})-\frac{\vec{v}}{m_{S}} \frac{d m_{S}}{d t}
$$

In eqn(4), $-\vec{g}$ represents the acceleration due to gravity, $\vec{B}$ is the terrestrial magnetic field, $q=Z e$ and $\vec{v}$ represents the velocity of the strangelet. These equations were solved by the $4^{\text {th }}$ order Runge Kutta Method for the set of initial conditions described above.

The results are shown in figs. $1-5$. Figure 1 shows the variation of Altitude with time, the zero of time being at $25 \mathrm{~km}$. The time required to reach a place which is about $3.6 \mathrm{~km}$ above the sea level (height of a typical north east Indian peak like 'Sandakphu', where an experiment to detect strangelets in cosmic rays using a large detector array is being set up [20]) is indicated in the figure. The next figure (Fig. 2) shows the variation of the mass of the strangelet with time and figure 3 shows the variation of the strangelet mass with altitude. It can be seen from the figures that the expected mass at the aforementioned altitude comes out to be about $340 \mathrm{amu}$ or so. Figure 4 shows the variation of the mean free time with altitude. Finally, figure 5 shows the variation of $\beta=v / c$ with time, showing the expected decrease of the speed with time, which also justifies the adequacy of the nonrelativistic treatment that we have applied.

In this letter, we have proposed a dynamical model of the propagation of strangelets through the atmosphere of the Earth. Although the model is based on simple assumptions, it is realistic enough to include the difference in the interaction process which is expected when SQM, and not ordinary nuclei, collide with the atmospheric nuclei. The effects of Earth's gravitational and magnetic fields are also included in the equations of motion so that meaningful information can be extracted directly from the resulting trajectory. The main conclusion of the model is that the exotic cosmic ray events with very small $Z / A$ ratios at mountain altitudes could result from SQM 


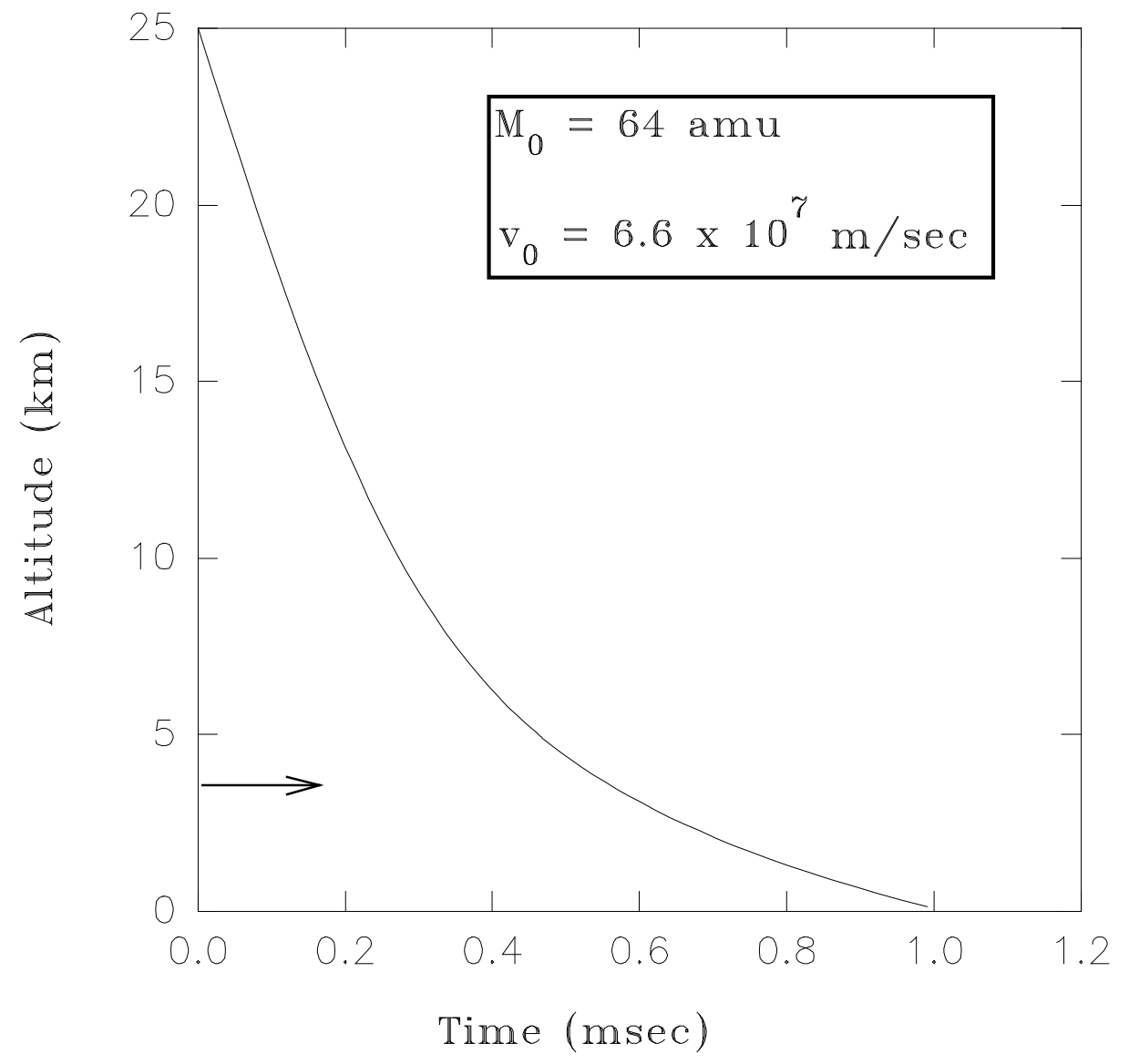

Figure 1: Variation of altitude with time. The arrow corresponds to an altitude of $3.6 \mathrm{~km}$ from the sea level. 


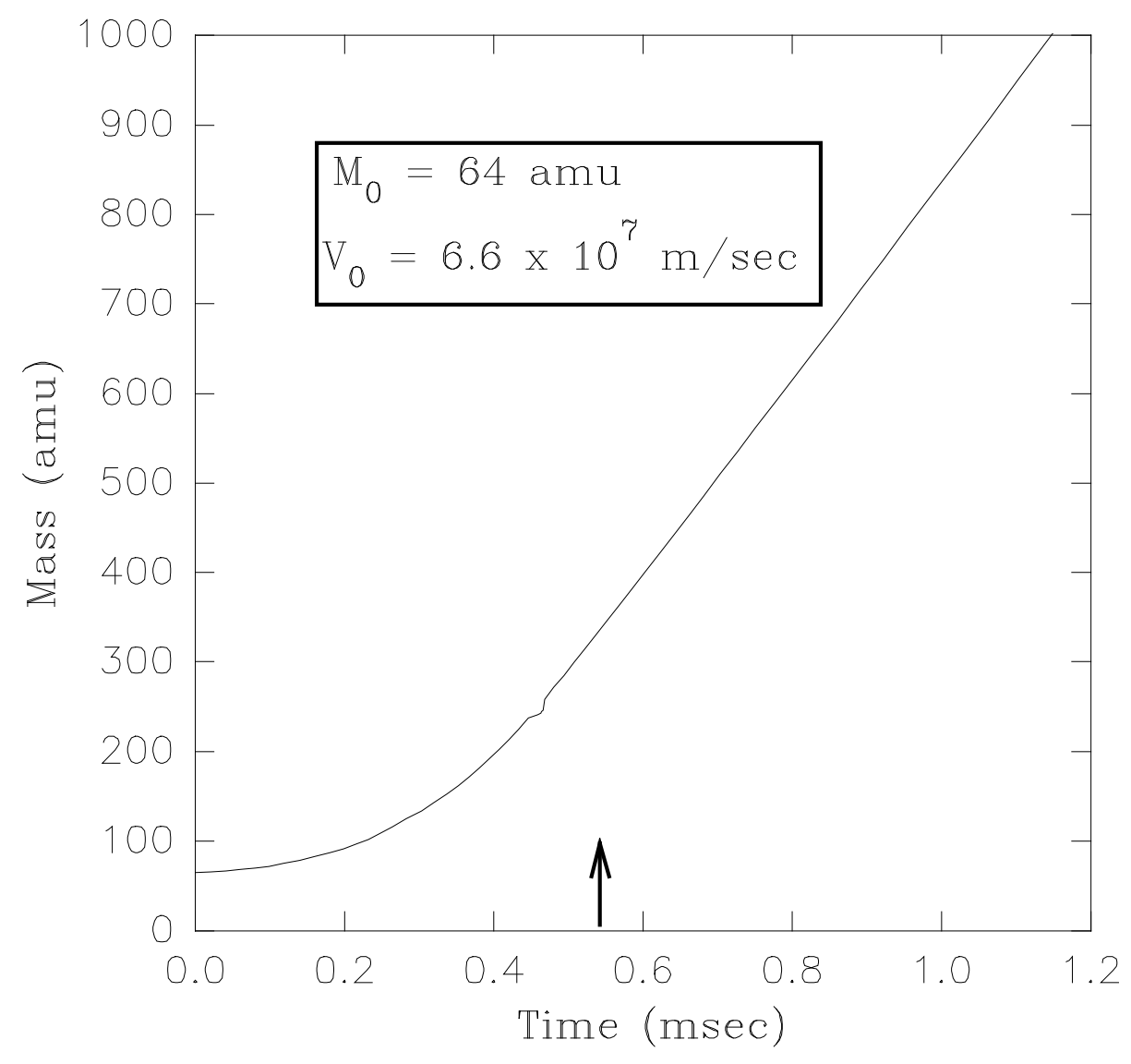

Figure 2: Variation of mass with time. The time indicated by the arrow is the time taken to reach an altitude $3.6 \mathrm{~km}$ from the sea level, starting from $25 \mathrm{~km}$. 


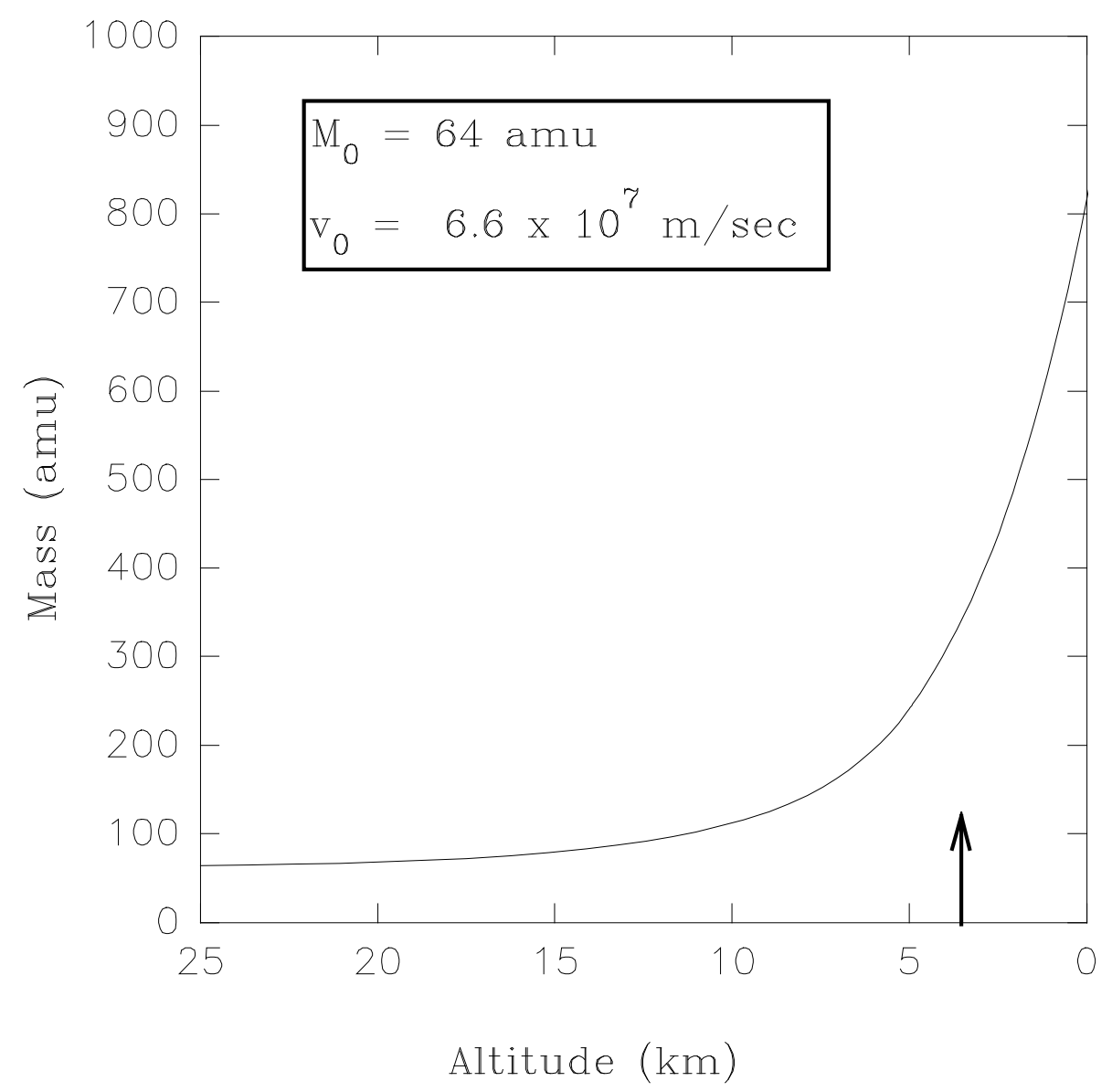

Figure 3: Variation of mass with altitude. The arrow corresponds to an altitude of $3.6 \mathrm{~km}$ from the sea level. 


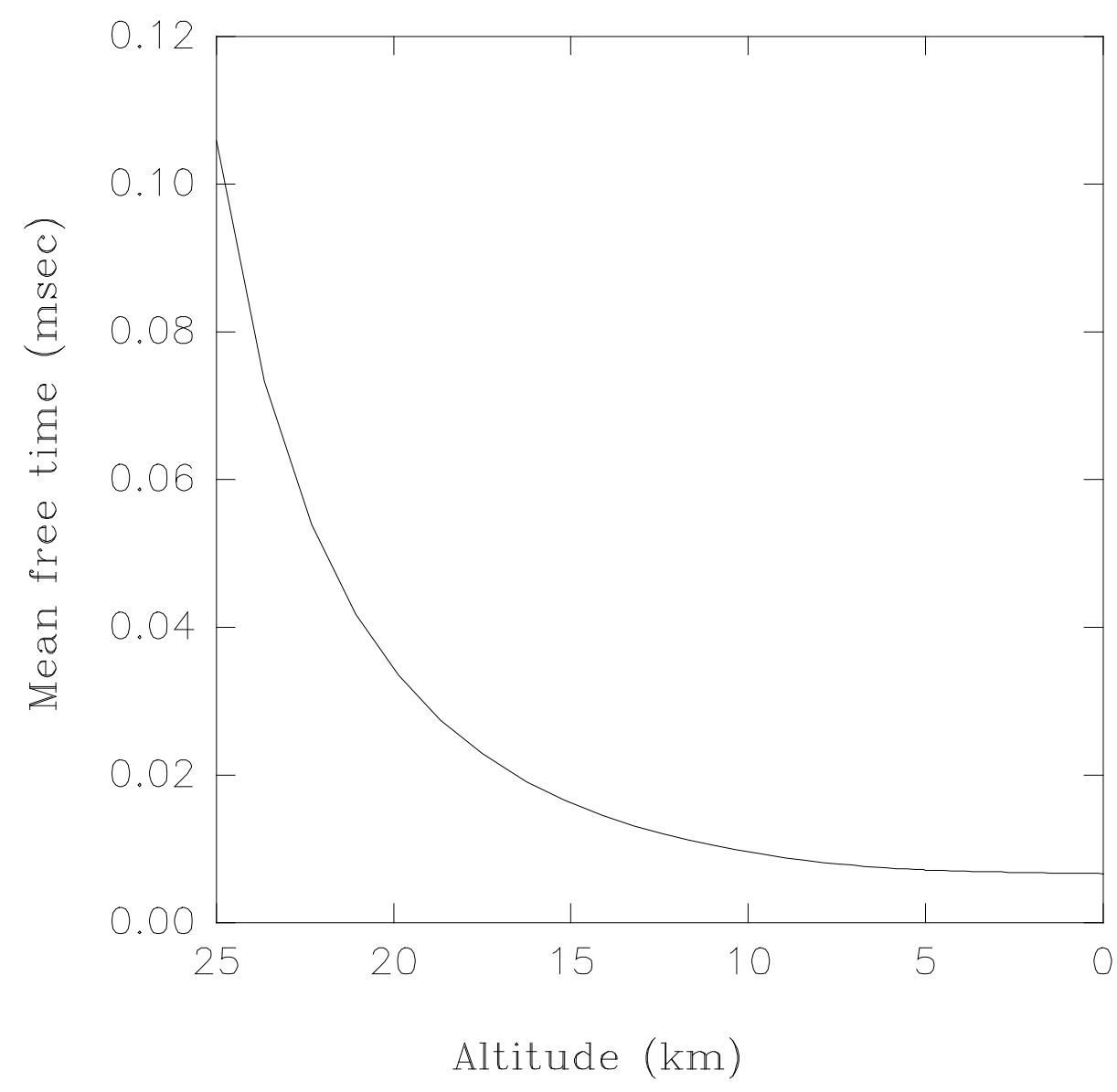

Figure 4: Change of mean free time with altitude. 


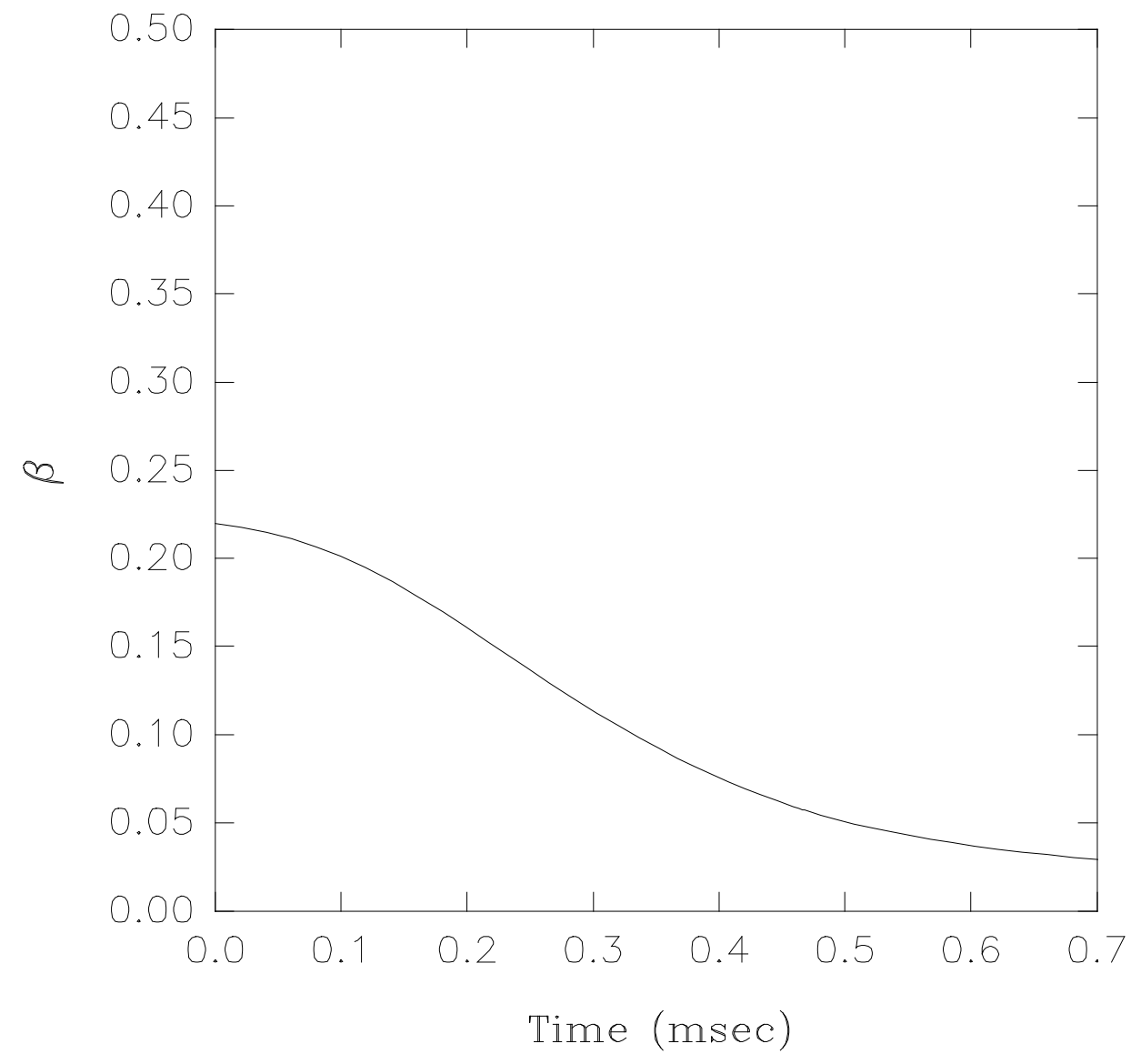

Figure 5: Change of $\beta=v / c$ with time. 
droplets (strangelets) which need not be too large initially. Thus the flux of strangelets in the cosmic rays may indeed be appreciable enough to make their detection by a large area detector at mountain altitudes a real possibility.

The works of SB and SKG were supported in part by the Council of Scientific \& Industrial Research, Govt. of India, New Delhi.

\section{References}

[1] E. Witten, Phys. Rev. D30, 272 (1984)

[2] J. Alam, S. Raha and B. Sinha, Ap. J. (in press)

[3] E. Farhi and R. L. Jaffe, Phys. Rev. D30, 2379 (1984)

[4] E. Farhi and R. L. Jaffe, Phys. Rev. D30, 1307 (1986)

[5] E. P. Gilson and R. L. Jaffe, Phys. Rev. Lett 71, 332 (1993)

[6] M. G. Mustafa and A. Ansari, Phys. Rev. D53, 5136 (1996)

[7] M. G. Mustafa and A. Ansari, Phys.Rev. C55, 2005 (1995)

[8] Jes Madsen,astro-ph 9809032; to appear in Hadrons in Dense Matter and Hadrosynthesis, Lecture Notes in Physics, Springer Verlag, Heidelberg.

[9] M. Kasuya et al., Phys. Rev. D47, 2153 (1993)

[10] M. Ichimura et al., Nuovo Cim. A106, 843 (1993)

[11] T. Saito, Proc.24 th ICRC Rome 1, 898 (1995)

[12] O. Miyamura, Proc.24 $4^{\text {th }}$ ICRC Rome 1, 890 (1995)

[13] J. N. Capdeville et al., Proc.24 ${ }^{\text {th }}$ ICRC Rome 1, 910 (1995) 
[14] G. Wilk and Z. Wlodarczyk, J. Phys. G22, L105 (1996)

[15] E. Gadysz-Dziadus and Z.Wlodarczyk, J. Phys. G23, 2057 (1996)

[16] G. Wilk and Z. Wlodarczyk, Nucl. Phys. (Proc. Suppl.) 52B, 215 (1997)

[17] G. Wilk and Z. Wlodarczyk, hep-ph 9606401

[18] Fundamental Formulas of Physics, Vol. 2, Donald H. Menzel (Ed), p. 560, Dover Publications, New York (1960)

[19] J. Gosset et al., Phys. Rev. C16, 629 (1977)

[20] Science at High Altitudes, S. Raha, P. K. Ray and B. Sinha (Eds.), Allied Publishers, New Delhi (1998) 